\title{
Role of echocardiography in differential diagnosis of broad complex tachycardia
}

\author{
CHRISTOPHER WREN, RONALD W F CAMPBELL, STEWART HUNTER \\ From the Freeman Hospital, Newcastle upon Tyne
}

SUMMARY It is not always easy to distinguish between supraventricular tachycardia with aberration and ventricular tachycardia by electrocardiographic analysis alone. $M$ mode echocardiography can often help by providing direct or indirect evidence of the relation between atrial and ventricular contraction. Sixteen consecutive patients with spontaneous sustained broad QRS complex tachycardia with heart rates of 120-225 beats/minute were examined. Echocardiographic evidence of 1:1 conduction was seen in three cases and 2:1 atrioventricular conduction in one (all four had supraventricular tachycardia, confirmed by intracardiac electrocardiography in three). Evidence of retrograde block was seen in 12 (all had ventricular tachycardia, with electrophysiological confirmation in 10). Satisfactory views of the mitral valve were obtained in all patients.

Patients with ventricular tachycardia had a variable mitral valve opening time (range 42-110\%) compared with those who had supraventricular tachycardia $(9-15 \%)$. Aortic root and left atrial views gave direct evidence of atrial contraction in three cases, and subcostal right atrial wall views were diagnostic in four of five cases. Seven patients with ventricular tachycardia had been wrongly diagnosed elsewhere as having supraventricular tachycardia. This study confirms that echocardiography is a simple and rapid aid to accurate diagnosis in patients with broad QRS complex tachycardia.

It may be difficult to decide whether a broad complex tachycardia is supraventricular or ventricular in origin. Examination of the surface 12 lead electrocardiogram may provide an accurate diagnosis. ${ }^{12}$ Clinical examination may also be helpful ${ }^{3}$ if simultaneous observation of the jugular venous pulse and palpation of the arterial pulse show evidence of associated or dissociated atrial contraction.

When the diagnosis is in doubt after clinical examination and analysis of the electrocardiogram, the choice lies between assessing the response (physiological or pharmacological) to treatment and invasive investigation.

$M$ mode echocardiography has been shown to be a useful investigation in this situation, mainly by providing non invasive evidence of atrioventricular association or dissociation. ${ }^{4-6}$ Various echocardiographic observations and variables have been suggested. ${ }^{467}$ We set out to examine which views, fea-

Requests for reprints to Dr C Wren, Ward 23, Freeman Hospital, Newcastle upon Tyne NE7 7DN.

Accepted for publication 29 April 1985 tures, and measurements would be useful in the diagnosis of broad complex tachycardias and to assess the technical problems of obtaining good quality recordings.

\section{Patients and methods}

All patients with regular broad complex tachycardias seen in a 16 month period in 1982-83 were eligible for the study whether or not the diagnosis was apparent from the initial electrocardiogram.

We studied 16 patients (12 male) aged 11-82 years. Thirteen had ischaemic heart disease, and in five the arrhythmia was associated with recent or acute infarction. Of the others, one had an ostium primum atrial septal defect, one hypertrophic cardiomyopathy, and one no identifiable heart disease.

Seven patients were referred from other hospitals after initial treatment of their arrhythmias had been unsuccessful. Nine patients had already had antiarrhythmic drugs by the time of examination, and in seven (including six referred patients) the initial treatment had been inappropriate because the correct diagnosis had not been made. 
We set out to record parasternal $M$ mode traces of the mitral valve and of the aortic valve and left atrium in all cases, and in the last five patients we also performed a subcostal examination. All M mode scans were derived from the sector scan.

Recordings good enough to establish the relation between atrial and ventricular contraction were obtained in all patients. Some patients in the group were poor subjects for echocardiographic examination and many were old or ill. Echocardiography is difficult in this situation, and if good quality recordings were not quickly available from all parts of the heart and in all views we did not persist with the examination because urgent treatment was often considered necessary. In two patients the episodes of arrhythmia ended spontaneously and the examination was incomplete.

All patients had a standard 12 lead electrocardiogram and a rhythm strip. The echocardiograms were recorded at $100 \mathrm{~mm} / \mathrm{s}$ with a simultaneous electrocardiogram and were obtained with a Toshiba SSH-10A phased array scanner or an Advanced Technology Laboratories MK 300C mechanical scanner, both with $3 \mathrm{MHz}$ probes. Where possible, 20 consecutive cycles of the mitral valve and the aortic valve were recorded. Manyari et al first described the pattern of movement of the mitral and aortic valves in supraventricular and ventricular tachycardia. ${ }^{4}$ In the light of their report we measured the following in our patients: cycle length; duration of mitral valve opening; and duration of aortic valve opening. The percentage variability of the mitral and aortic opening times was calculated as: $100 \times($ maximum-minimum value)/minimum value. In addition, the mitral valve, aortic valve, posterior aortic wall, left atrial posterior wall, and subcostal right atrial wall were examined for evidence of independent or associated atrial activity ("A" waves).

In four patients the diagnosis was made confidently from the electrocardiogram and the response to treatment. In two patients echocardiography was performed during subsequent electrophysiological stimulation of the same arrhythmias because they had been too ill on admission for inclusion in the study. Twelve patients underwent electrophysiological study, usually a limited procedure to confirm the diagnosis and to terminate the arrhythmia by means of programmed stimulation.

\section{Results}

Satisfactory views of the mitral valve were obtained in all patients. The aortic valve was seen well enough for measurement of the opening time in only four patients, and the posterior wall of the aorta provided diagnostic information in another two. The left atrial posterior wall was seen clearly in eight patients, but in only one case were definite atrial contractions seen. Subcostal echocardiography was performed in the last five patients and good views of the right atrial wall were obtained in four. These results are summarised in the Table.

Twelve patients were found to have ventricular tachycardia with heart rates between 132 and 225 beats/min (mean (SD) 165 (25)). Eleven of the 12 had ischaemic heart disease. The diagnosis of ventricular tachycardia was confirmed by electrophysiological study in 10 cases. Of the 12 patients, 10 had evidence of ventriculoatrial dissociation and two had retrograde Wenckebach block. In two cases the admission electrocardiogram showed 2:1 ventriculoatrial conduction, but there was retrograde block by the time the echocardiogram was performed, the change in one case being in response to carotid sinus massage. No ventricular tachycardia with 1:1 retrograde conduction was seen.

Four patients had supraventricular tachycardia with heart rates of $120-190$ beats/min (mean (SD) 158 (34)). There was $1: 1$ atrioventricular conduction in three, confirmed by electrophysiological study in two. The fourth patient had atrial flutter with 2:1 conduction. Two patients with supraventricular tachycardia had aberrant conduction during the tachycardia, and the other two had an underlying bundle branch block when they returned to sinus rhythm.

The echocardiogram correctly identified the relation between atrial and ventricular contraction in all patients, but in the case of $1: 1$ conduction it could not distinguish between anterograde and retrograde conduction. The variability of mitral opening times in ventricular tachycardia was $42-110 \%$ and in supraventricular tachycardia it was $9-15 \%$ (Fig. 1). Aortic valve opening time in ventricular tachycardia was $32 \%, 33 \%$, and $54 \%$ in three cases and only one value was obtained in supraventricular tachycardia (16\%).

Independent " $A$ " waves on the anterior mitral valve leaflet have been described in ventricular tachycardia ${ }^{4}$ but were seen in only four cases in this series, probably because heart rates in our group were higher than those reported previously. " $A$ " waves

Table Success rates of various echocardiographic views. Figures are numbers of patients

\begin{tabular}{lcc}
\hline & Clearly seen & Diagnostic $^{\star}$ \\
\hline PS mitral valve & $16 / 16 \dagger$ & $16 / 16$ \\
PS aortic valve & $4 / 16 \dagger$ & $4 / 16$ \\
PS aortic wall & $9 / 16$ & $2 / 16$ \\
PS left atrial wall & $8 / 16$ & $1 / 16$ \\
SC right atrial wall & $4 / 5$ & $4 / 5$
\end{tabular}

$\star$ Gave definite evidence of relation between atrial and ventricular contraction.

tSeen clearly enough for accurate measurement.

PS, parasternal; SC, subcostal. 


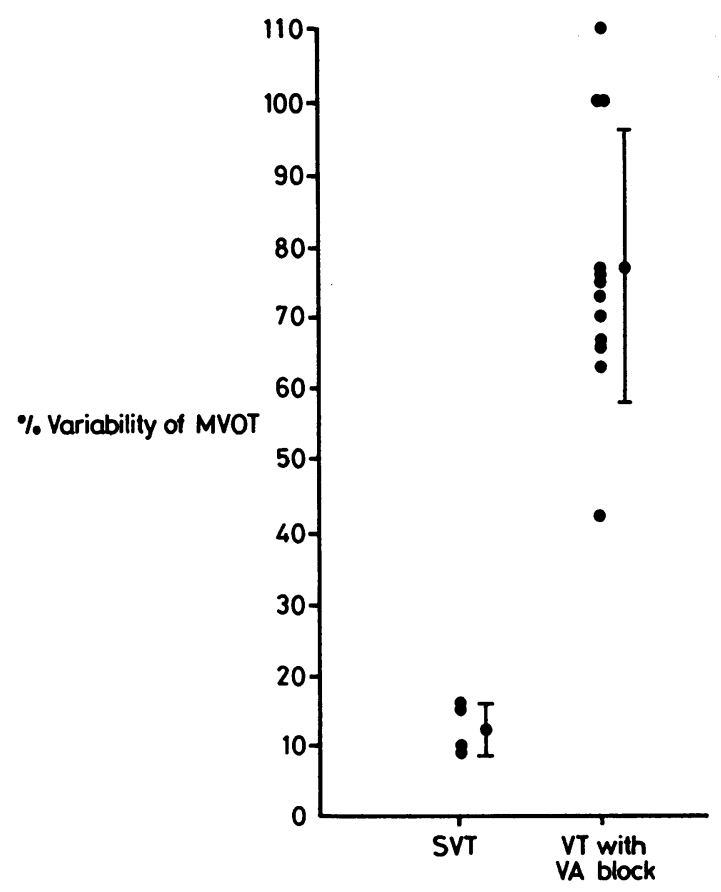

Fig. 1 Range of variability of mitral valve opening time (MVOT) in patients with supraventricular tachycardia (SVT) or ventricular tachycardia (VT) with ventriculoatrial (VA) block. Bars denote mean (1 SD). were not seen on the aortic valve or left ventricular recordings in any patient.

Figure 2 shows the pronounced variability of mitral opening times seen in ventricular tachycardia, and this was easily distinguished from that seen in 1:1 conduction (Fig. 3). Mitral opening was also constant in 2:1 atrioventricular conduction. Figure 4 shows the variable aortic valve opening time seen in ventricular tachycardia, whereas aortic opening in supraventricular tachycardia is regular.

Evidence of dissociated atrial activity was occasionally seen on the posterior wall of the aorta in ventricular tachycardia and when present was diagnostic (Fig. 5). Echocardiography of the left atrial wall was generally disappointing as, even when it was seen clearly and there was other echocardiographic evidence of atrial contraction, "A" waves were not seen (Fig. 5).

Subcostal views were diagnostic in four patients. The recordings were taken from the right atrial wall just above the tricuspid valve ring with the $M$ mode beam being positioned on the sector scan. It was apparent from examining both cross sectional and $M$ mode recordings that movement transmitted from ventricular contraction produced low amplitude movement or vibration of the right atrial wall in time with the QRS complex on the electrocardiogram. Atrial contractions appeared as larger amplitude discrete posterior movements.

Subcostal examination showed ventricular tachycardia with retrograde block in one patient (Fig. 6) and ventricular tachycardia with retrograde Wenckebach conduction in another. ${ }^{8} \mathrm{~A}$ third patient had

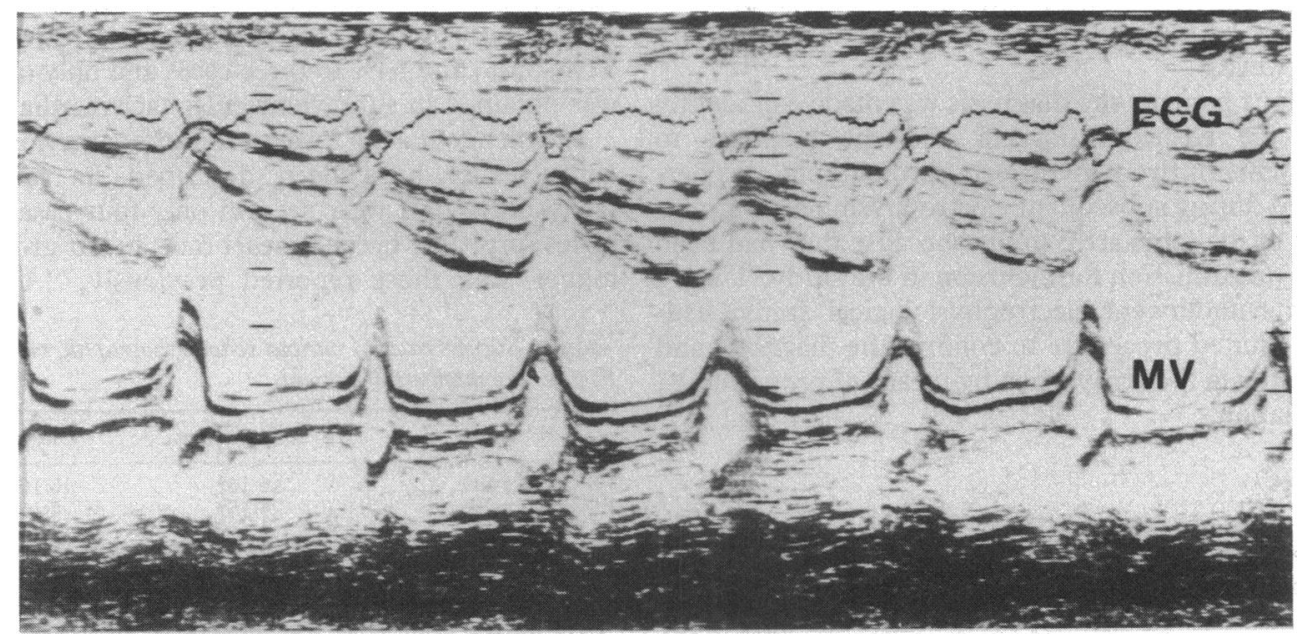

Fig. 2 Parasternal $M$ mode echocardiographic view of the mitral valve (MV) in a patient with ventricular tachycardia. There is pronounced beat to beat variability of mitral opening time. 


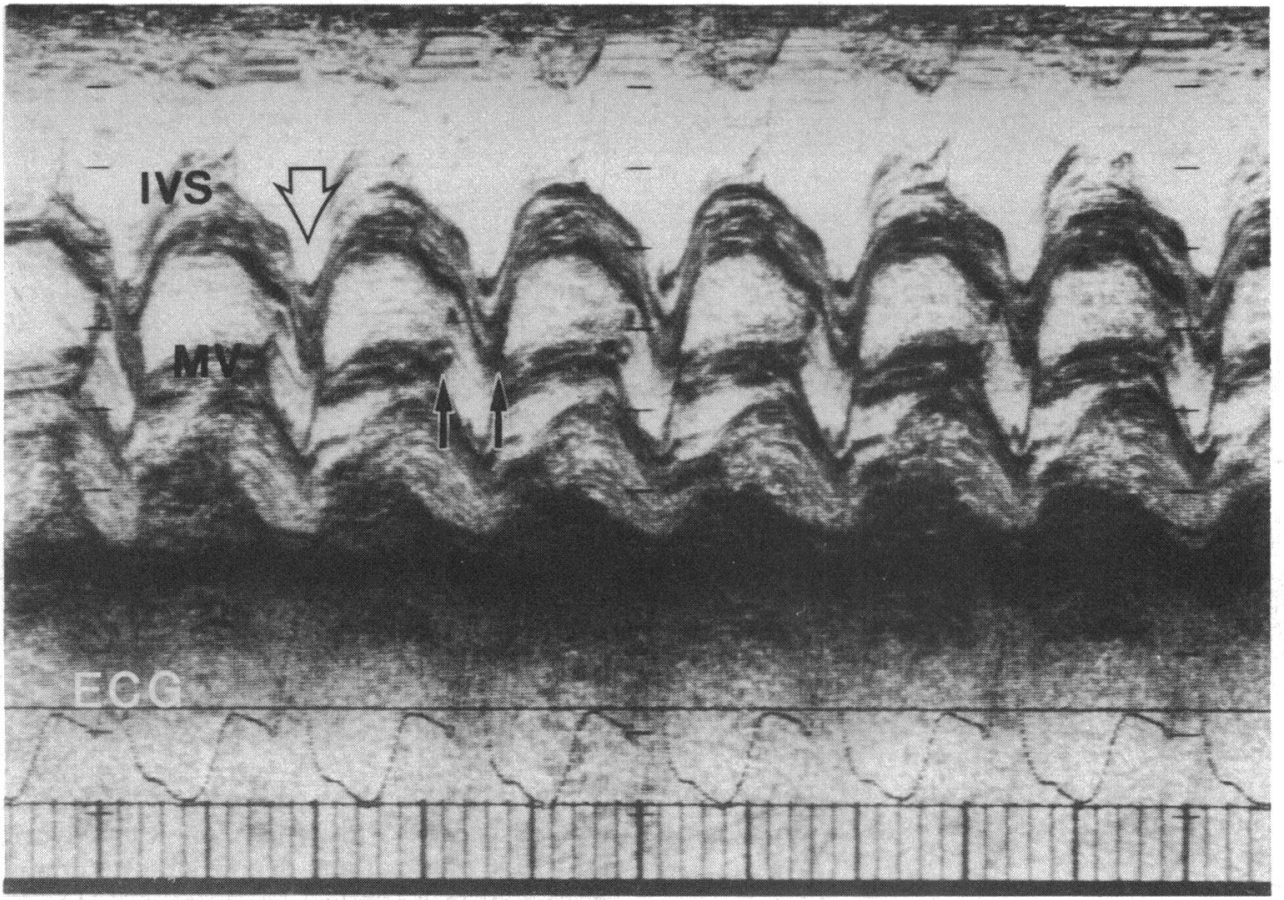

Fig. 3 Parasternal view of the mitral valve (MV) showing the regular mitral opening (arrows) seen with $1: 1$ conduction. The diagnosis at electrophysiological study was supraventricular tachycardia. Note the dilated right ventricle and reversed movement (open arrow) of the interventricular septum (IVS) caused by a coexisting ostium primum atrial septal defect.

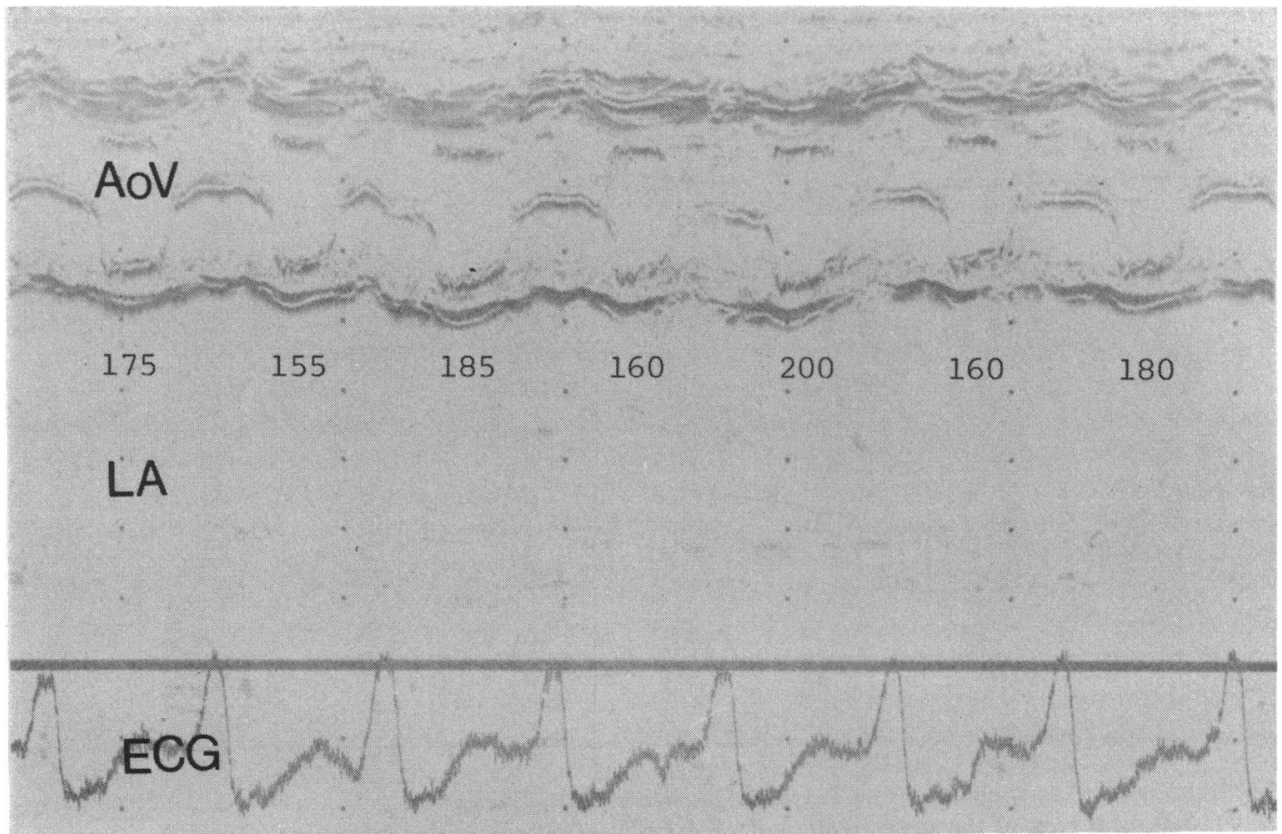

Fig. 4 Parasternal view of the aortic valve (AoV) and left atrium (LA) in ventricular tachycardia. The duration of aortic valve opening varies from beat to beat. Aortic valve opening times are given in ms. 


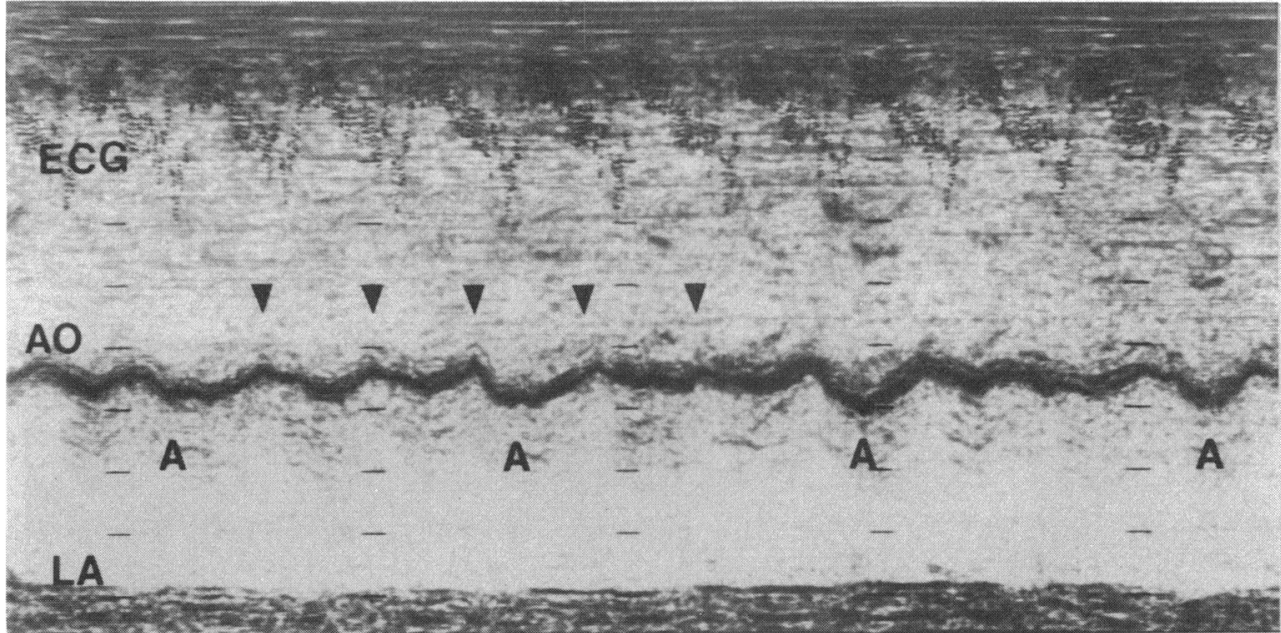

Fig. 5 Parasternal view through aorta (AO) and left atrium (LA) showing evidence of clearly dissociated atrial activity $(A)$ on the posterior wall of the aorta. The arrows show aortic movement related to ventricular contraction. The electrocardiogram (ECG) is of poor quality. Note the absence of contraction of the posterior wall.

supraventricular tachycardia with $1: 1$ conduction (Fig. 7) and the fourth had atrial flutter with 2:1 conduction.

\section{Discussion}

Echocardiographic differentiation between supraventricular and ventricular tachycardia depends on iden- tifying the relation between atrial and ventricular contraction. Patients with broad complex tachycardia and retrograde block of any degree almost certainly have ventricular tachycardia. The retrograde block can be demonstrated directly by showing dissociated atrial activity on the left or right atrial walls (Figs. 5 and 6) or indirectly by showing irregular mitral valve opening (Fig. 2). The echocardiographic appearance of the

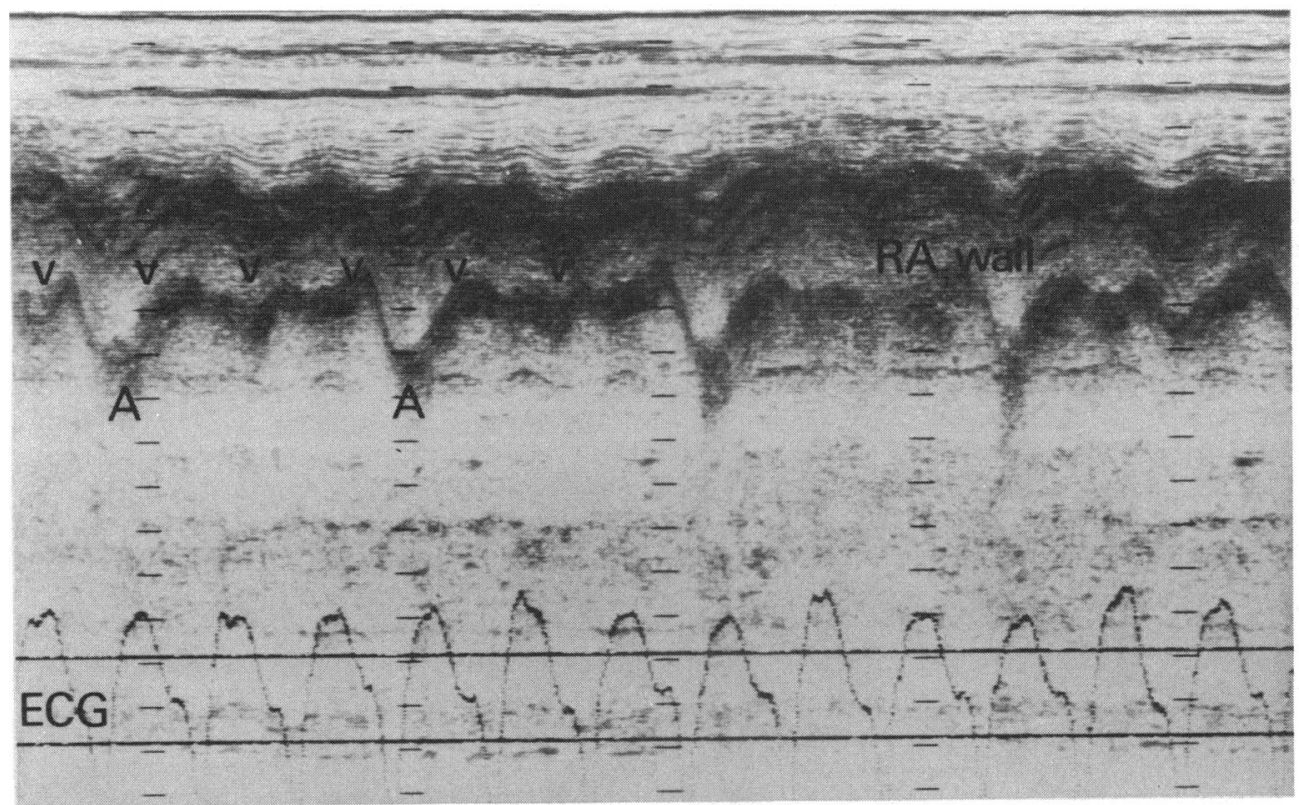

Fig. 6 Subcostal view of the right atrial wall ( $R A$ wall). The transmitted movement related to ventricular contraction $(V)$ coincides with the electrocardiogram (ECG). Dissociated atrial contraction (A) is shown as clear posterior movement. Reproduced with permission of American Heart fournal. ${ }^{8}$ 


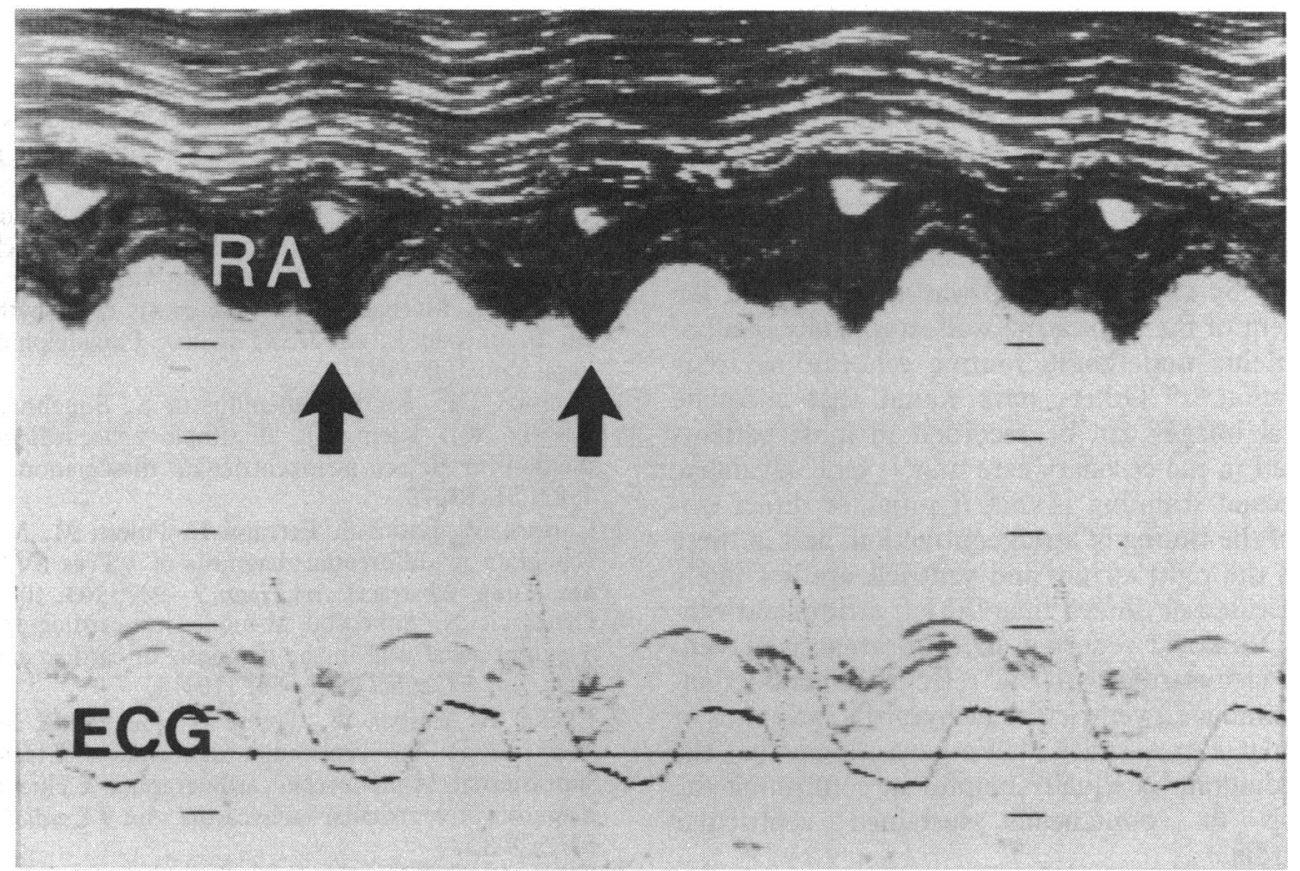

Fig. 7 Subcostal view of right atrial wall (RA) in supraventricular tachycardia in the same patient as in Fig. 3. There is a 1:1 relation between atrial contraction (arrows) and the QRS on the electrocardiogram (ECG).

mitral valve in broad QRS complex tachycardias was described by Manyari et al. ${ }^{4}$ They found a clear distinction in the variability of mitral valve opening between anterograde or retrograde conduction and atrioventricular dissociation. Their values of less than $22 \%$ variability of mitral opening and less than $12 \%$ variability of aortic opening with $1: 1$ conduction, and 68-129\% mitral variability and $41-175 \%$ aortic variability in ventricular tachycardia with retrograde block accord with our results. They had only eight patients, however, with pacing simulated arrhythmias in three patients, and a maximum heart rate of 143 beats/min. Our study shows that variabilty of the time of mitral opening can clearly identify the relation between atrial and ventricular contraction in unselected patients with spontaneous arrhythmias with heart rates up to 225 beats per minute.

When anterograde block is present the diagnosis must be supraventricular tachycardia. In the event of 1:1 conduction the echocardiogram cannot at present be used to distinguish between supraventricular tachycardia and ventricular tachycardia. It may eventually be possible to tell whether conduction is anterograde or retrograde by timing atrial and ventricular contraction on the echocardiogram but present experience does not allow this.

Fortunately, 1:1 retrograde conduction is uncom- mon in spontaneous sustained ventricular tachycardia with fast heart rates, ${ }^{9}$ and so echocardiographic confirmation of $1: 1$ conduction means that supraventricular tachycardia is the likely diagnosis. Obviously, the echocardiogram will not be used in isolation and electrocardiographic features will be taken into account as well.

The patients in this study may well be an atypical group as several were referred when they did not respond to initial treatment. In most cases they had been treated for supraventricular tachycardia, whereas we proved the presence of ventricular tachycardia in all the referred patients. Retrograde conduction may have been modified by the drugs they had received, and this may explain why no ventricular tachycardia with 1:1 retrograde conduction was seen.

Views of the mitral valve were of great value in all our patients, whereas aortic valve and left atrial views were less often helpful. Many of our patients had ischaemic heart disease with impaired left ventricular function and left atrial enlargement. This may explain why we did not see " $A$ " waves on the posterior wall of the left atrium (Fig. 5) but did see them in the right atrium (Fig. 6). Other workers have found left atrial echocardiograms useful in the diagnosis of broad QRS complex tachycardias. ${ }^{7}$

$M$ mode recordings of the left ventricle were gener- 
ally of poor quality and were unhelpful. This was not surprising as most patients had left ventricular disease and ventricular contraction during ventricular tachycardia is incoordinate and is much reduced. " $A$ " waves were not seen on the left ventricular traces in any patient.

The echocardiographic characteristics of various arrhythmias seen by subcostal scanning were described by Drinković, who was able to record the movement of the right atrial wall subcostally in all of 260 patients undergoing routine echocardiographic examination. ${ }^{610}$ Others have found that adequate subcostal images can be recorded in most patients examined in the coronary care unit. ${ }^{11}$ One advantage of subcostal scanning is that it provides direct evidence of the timing of atrial contraction, and in these patients the right atrium and ventricle are less likely to be diseased or dilated than the left atrium and ventricle. Drinkovic reported the appearances of ventricular tachycardia with 1:1 retrograde conduction, pacing simulated ventricular tachycardia, and runs of ventricular extrasystoles. ${ }^{10}$ We have shown that the echocardiogram is equally helpful in confirming the diagnosis in spontaneous sustained ventricular tachycardia.

Although all the $M$ mode recordings in this series were derived from the sector scan, it is possible to obtain satisfactory results with an $M$ mode machine alone. ${ }^{4610}$ This brings the technique within the capability of many more hospitals. We feel that echocardiography is of great value when the electrocardiogram alone cannot distinguish between supraventricular and ventricular tachycardia. With increasing experience of the technique and its interpretation the echocardiogram could become a standard investigation in the diagnosis of broad complex tachycardias.

\section{References}

1 Wellens HJJ, Bär FWHM, Lie KI. The value of the electrocardiogram in the differential diagnosis of a tachycardia with a widened QRS complex. Am $\mathcal{F}$ Med 1978; 64: 27-33.

2 Dancy M, Scaffidi L, Camm AJ, Ward D. Ventricular tachycardia misdiagnosed as supraventricular tachycardia [Abstract]. Br Heart f 1985; 53: 98-9.

3 Bigger JT. Mechanisms and diagnosis of arrhythmias. In: Braunwald E, ed. Heart disease. Philadelphia: WB Saunders, 1980: 647-8.

4 Manyari DE, Ko P, Gulamhusein S, Boughner DR, Kostuk WJ, Klein GJ. A simple echocardiographic method to detect atrioventricular dissociation. Chest 1982; 81: 67-73.

5 Curione M, Latini S, Ferranti E, Puletti M. M-mode echogram in differential diagnosis of VT vs SVT with aberration |Abstractl. Am Heart F 1982; 103: 1084.

6 Drinković N. Subcostal M-mode echocardiography of the right atrial wall in the diagnosis of cardiac arrhythmias. Am ₹ Cardiol 1982; 50: 1104-8.

7 Rückel A, Kasper W, Treese N, Henkel B, Pop T, Meinertz T. Atrioventricular dissociation detected by suprasternal M-mode echocardiography: a clue to the diagnosis of ventricular tachycardia. Am $\mathcal{F}$ Cardiol 1984; 54: 561-3.

8 Wren C. Subcostal echocardiography in ventricular tachycardia. Am Heart $\mathcal{F}$ 1984; 108: 1579-82.

9 Puech P. Diagnosis of ventricular tachycardias. In: Masoni A, Alboni P, eds. Cardiac electrophysiology today. London: Academic Press, 1982: 273-90.

10 Drinković N. Subcostal $M$-mode echocardiography of the right atrial wall for differentiation of supraventricular tachyarrhythmias with aberration from ventricular tachycardia. Am Heart f 1984; 107: 326-31.

11 Gibson RS, Bishop HL, Stamm RB, Crampton RS, Beller GA, Martin RP. Value of early two dimensional echocardiography in patients with acute myocardial infarction. Am f Cardiol 1982; 49: 1110-9. 